\title{
Resección laparoscópica de schwannoma gástrico. Reporte de caso
}

\section{Laparoscopic resection of gastric schwannoma. Case report}

\section{Ressecção laparoscópica do schwannoma gástrico. Relatório de caso}

\author{
doi) http://dx.doi.org/10.35954/SM2020.39.1.5 \\ Martín Varela Vega ${ }^{a}$ (i) https://orcid.org/0000-0003-2376-2844 \\ Leticia Barro a (D) https://orcid.org/0000-0001-6940-0342 \\ Micaela Alvariza a (D) https://orcid.org/0000-0002-8126-2250 \\ Agustina Da Rosa a (D) https://orcid.org/0000-0002-7313-1306 \\ Santiago Cubas a (D) https://orcid.org/0000-0001-6026-5316 \\ Gerardo Beraldo a (D) https://orcid.org/0000-0002-3311-160X \\ Pablo Santiago a (D) https://orcid.org/0000-0002-7733-8291
}

(a) Sección Cirugía Esófago Gástrica. Departamento de cirugía general. Hospital Central de las Fuerzas Armadas.

\section{RESUMEN}

Los Schwannomas son tumores mesenquimáticos que se dan raramente en el estómago. Se originan en las células de Schwann o células de la vaina periférica de los nervios del plexo de Meissner y Auerbach. Son poco sintomáticos, y la clínica viene dada por el crecimiento, que genera síntomas compresivos, o por elementos de hemorragia digestiva alta, al ulcerar la mucosa.

El algoritmo de estudio comprende la endoscopía digestiva alta, la ecoendoscopía, tomografía computada y tránsito contrastado digestivo.

El tratamiento es quirúrgico, y el análisis anatomopatológico de la pieza, con el recurso de la inmunohistoquímica, permiten confirmar el diagnóstico. El pronóstico es excelente, ya que son generalmente benignos.

PALABRAS CLAVE: Neurilemoma; Schwannoma.

\begin{abstract}
Schwannomas are mesenchymal tumors that rarely occur in the stomach. They originate in Schwann cells or cells of the peripheral sheath of the Meissner and Auerbach plexus nerves. They are not very symptomatic, and the clinic is given by the growth, which generates compressive symptoms, or by elements of upper digestive hemorrhage, when the mucosa is ulcerated.

The study algorithm includes upper endoscopy, echoendoscopy, computed tomography and contrasting digestive transit.
\end{abstract}


The treatment is surgical, and the anatomopathological analysis of the piece, with the resource of immunohistochemistry, allows confirming the diagnosis. The prognosis is excellent, since they are generally benign.

KEY WORDS: Neurilemmoma; Schwannoma.

\section{RESUMO}

Os Schwannomas são tumores mesenquimais que raramente ocorrem no estômago. São originários de células de Schwann ou células da bainha periférica dos nervos Meissner e Auerbach plexus. Não são muito sintomáticos, e a clínica é dada pelo crescimento, que gera sintomas compressivos, ou por elementos de hemorragia gastrointestinal superior, quando a mucosa é ulcerada.

O algoritmo do estudo inclui endoscopia superior, econdoscopia, tomografia computorizada e trânsito digestivo contrastante.

O tratamento é cirúrgico, e a análise anatomopatológica da peça, com o recurso da imuno-histoquímica, permite confirmar o diagnóstico. O prognóstico é excelente, uma vez que são geralmente benignos.

PALAVRAS CHAVE: Neurilemoma; Schwannoma.

\section{INTRODUCCIÓN}

Los schwannomas son tumores mesenquimáticos que se dan raramente en el tubo digestivo (2 a $8 \%$ de los tumores mesenquimáticos digestivos). Dentro del intestino, la localización gástrica es la más frecuente, aunque constituyen el $0,2 \%$ del total de los tumores gástricos. Se originan en la vaina periférica de los nervios del plexo de Meissner y Auerbach, y son generalmente benignos. Hasta los primeros años de la década de los 80 , en la literatura era frecuente que este tipo de tumores fuera englobado dentro de los tumores del estroma gastro intestinal (GIST), o también como leiomiomas, tumores espinocelulares, o tumores derivados del músculo liso. Con el advenimiento de técnicas de inmunohistoquímica (IHQ) en los 80 , Mazur y colaboradores describieron la estirpe neurológica de algunos de estos tumores estromales, diferenciándolos del resto. Estos tumores, que con anterioridad habían sido catalogados como leiomiomas o leiomiosarcomas eran positivos para la inmunomarcación con la proteína S-100, un marcador para células derivadas del neuroectodermo (1). En el año 1988 Daimaru y colaboradores publicaron la primera serie con 24 tumores originados en las células de Schwann del tubo digestivo, pero que se diferenciaban inmunohistoquímica- mente de los neurofibromas y de los tumores del estroma gastro intestinal (GIST), por lo que propusieron para este tipo de tumores el nombre de "schwannomas del tracto gastrointestinal" (2).

Presentamos a continuación un caso clínico de diagnóstico y resolución en el servicio de Cirugía Esófago Gástrica del Hospital Central de las Fuerzas Armadas (H.C.FF.AA.).

Caso clínico: paciente de 75 años que consulta al gastroenterólogo por dolor epigástrico de tipo urente. La endoscopía digestiva muestra lesión submucosa de curvatura mayor gástrica de unos $5 \mathrm{~cm}$ de diámetro, con centro deprimido y ulcerado. Sin sangrado activo. Las biopsias endoscópicas informan mucosa gástrica sana. No contamos con biopsia por ecoendoscopía en el H.C.FF.AA. El estudio contrastado de esófago gastro duodeno muestra la lesión en cuerpo gástrico medio, sobre curvatura mayor (figura 1). La tomografía computada (TC) evidencia una lesión homogénea del estómago sin adenopatías regionales o metástasis a distancia (figura 2).

Conducta: con el planteo de tumor estromal de cuerpo gástrico (por frecuencia se plantea un GIST, y en segunda instancia un leiomioma) se decide cirugía resectiva por vía laparoscópica (figura 3). Se realiza una gastrectomía vertical atí- 
pica guiada sobre bujía orogástrica de $40 \mathrm{Fr}$ garantizando de ese modo un correcto calibre del estómago remanente (figura 4). La endoscopía intraoperatoria permite certificar que toda la lesión está entera en la pieza de resección. Cirugía sin incidentes, tolerando líquidos al día siguiente. Los controles radiológicos no evidenciaron fuga de contraste. Alta hospitalaria a las 48 horas. El estudio anátomo patológico y de IHQ de la pieza es concordante con un schwannoma gástrico. Muestra positividad difusa para sinaptofisina (S-100) y GFAP, siendo negativa para CD 117, desmina y angiomiolipoma (AML), lo que lo aleja del planteo de GIST y leiomioma (figuras 5 y 6 ).

\section{DISCUSIÓN}

Los schwannomas son tumores mesenquimáticos raros, generalmente benignos, clínicamente indistinguibles de otros tumores estromales. Pueden ser asintomáticos, y cuando presentan síntomas estos suelen ser vagos, hasta que una endoscopía del tubo digestivo superior logra diagnosticar un tumor submucoso. Son más comunes en mujeres, con una relación 4:1 (3). Pueden darse en solitario, o en el contexto de la Enfermedad de Von Recklinghausen (Neurofibromatosis tipo 1) (4). La clínica viene dada por el crecimiento, que genera síntomas compresivos, dispepsia o malestar abdominal. Se han descrito casos de estenosis gastroduodenal o disfagia en tumores de gran tamaño (5). Es relativamente frecuente la presencia de elementos de hemorragia digestiva alta, ya que pueden ulcerar la mucosa. De todos modos, más de la mitad de los casos son hallazgos en estudios endoscópicos o imagenológicos por otras causas, lo que habla del curso silente que toman frecuentemente.

La endoscopía permite topografiar la lesión, que es habitualmente submucosa, por lo que las biopsias superficiales no son de ayuda para realizar un diagnóstico de naturaleza diferenciando entre GIST, leiomiomas o schwannomas. La ecoendoscopía, cuando está disponible, es de utilidad para

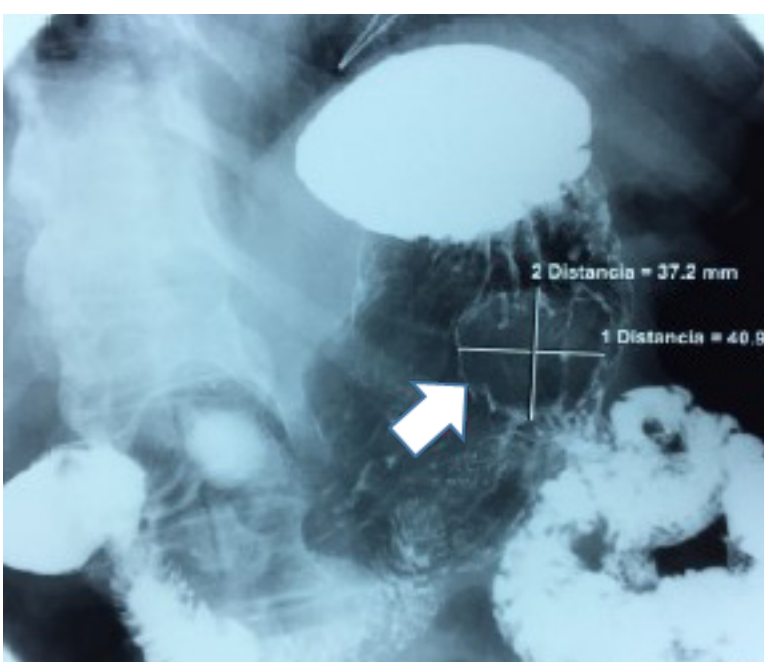

Figura 1. Tránsito de EGD con doble contraste. Se ve imagen de sustracción en cuerpo medio, sobre la curvatura mayor.

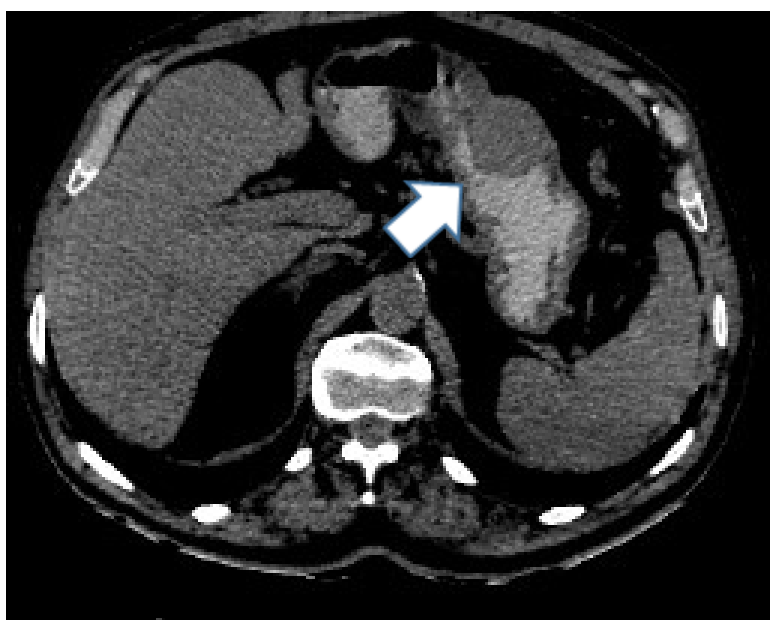

Figura 2. TC de abdomen (axial), lesión gástrica de unos $5 \mathrm{~cm}$ con crecimiento endoluminal.

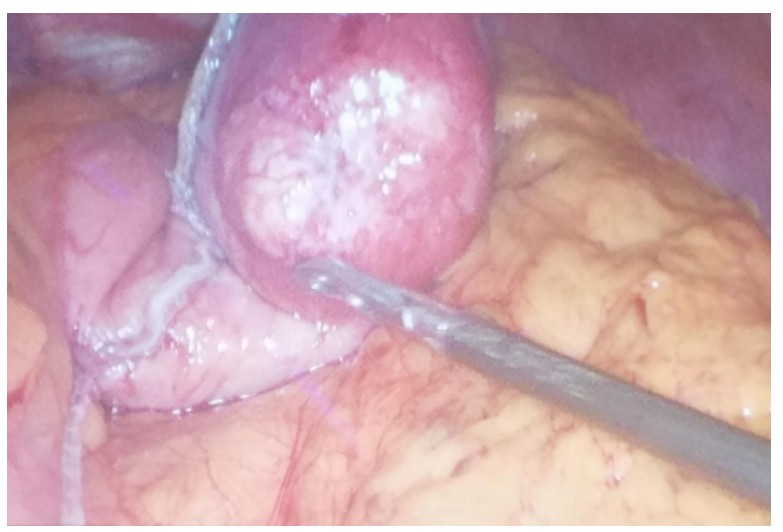

Figura 3. Cirugía: resección laparoscópica de la curvatura mayor, comprendiendo al tumor. 


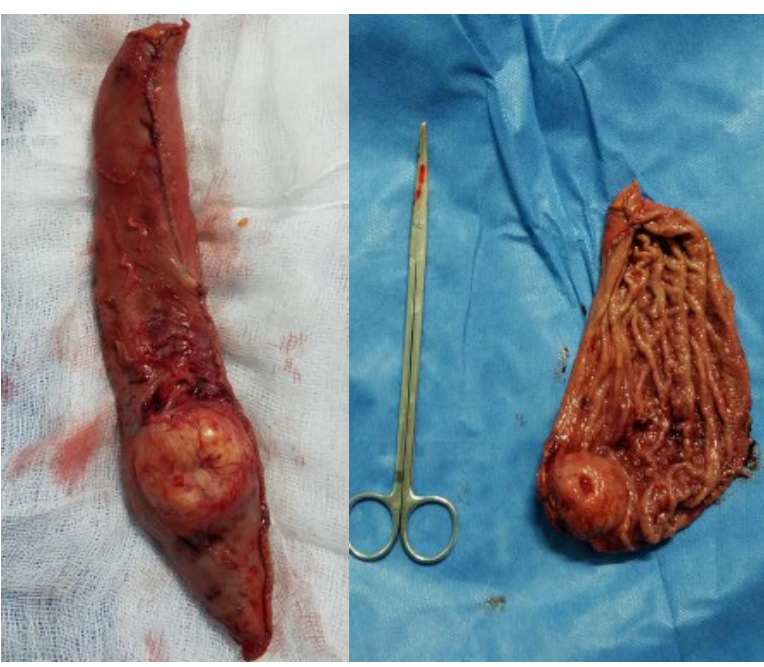

Figura 4. Pieza de resección. Tumor de unos $4 \mathrm{~cm}$ con centro ulcerado.

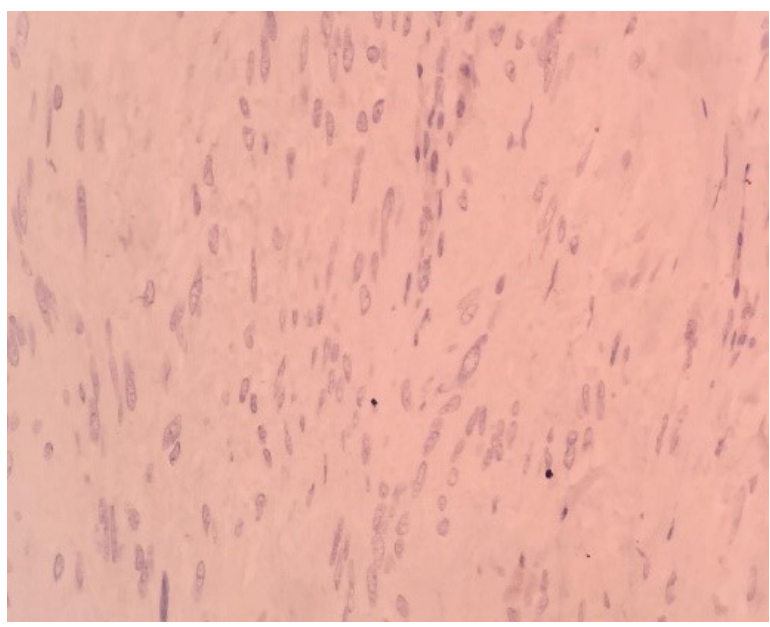

Figura 5. IHQ: CD 117 negativo.

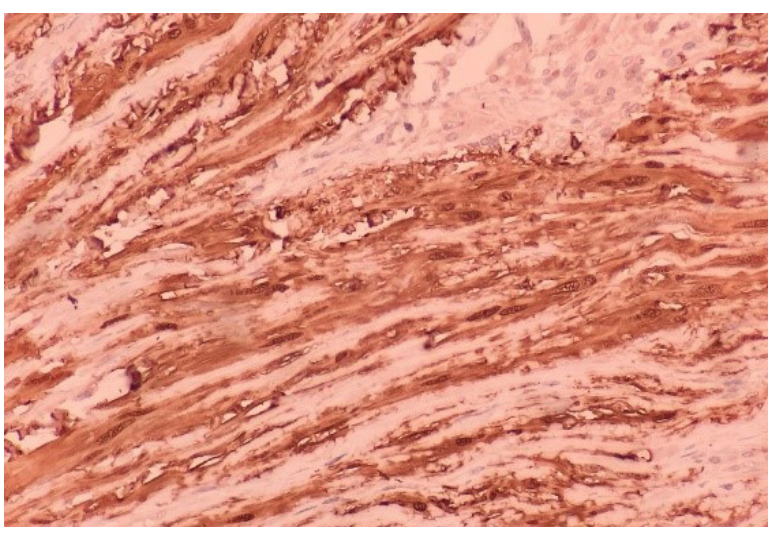

Figura 6. IHQ: S100 intensamente positivo. realizar una biopsia profunda de la lesión, que en muchos casos sella el diagnóstico (6).

La tomografía computada es de elección en la estadificación de cualquier tumor gástrico. En general suele verse una lesión redondeada y homogénea en el espesor de la pared gástrica, de carácter expansivo, no infiltrante, y sin adenomegalias ni imágenes de secundarismo (7). La localización precisa del tumor permite planificar la táctica quirúrgica, y en ese aspecto es valiosa la ayuda de los estudios contrastados con bario. Una vez realizado el diagnóstico de tumor gástrico submucoso, surge la indicación quirúrgica para resolver las complicaciones y tener una definición de la naturaleza del mismo mediante el estudio anátomo patológico e inmunohistoquímico de la pieza. Macroscópicamente son tumores de crecimiento expansivo, bien circunscritos, y usualmente recubiertos de mucosa sana, aunque en ocasiones esta última puede ulcerarse. De consistencia firme, elástica y color blanco rosado, pueden tener gran tamaño. A la microscopía pueden verse células fusiformes entrelazadas con abundante tejido colágeno y núcleos en empalizada (situación característica descrita por el patólogo uruguayo José Verocay en 1910), con alguna atipía nuclear y escasa o nula actividad mitótica (8).

Histológicamente en ocasiones los tumores estromales son indistinguibles entre si con la tinción hematoxilina eosina, por lo que se requieren técnicas de IHQ para diferenciar los GIST de leiomiomas o schwannomas. Los schwannomas son intensamente positivos para S-100 y GPAF, mientras que son negativos para CD 117 (característico de los GIST) y AML (característico de los leiomiomas) (9).

Una vez establecido el diagnóstico de schwannoma, el pronóstico es excelente, ya que la degeneración maligna es extremadamente infrecuente. La indicación de cirugía surge de la necesidad del análisis patológico de la pieza para descartar otras formas de tumores estromales más agresivos, y del hecho de que liberados a su evolución 
pueden dar complicaciones vinculadas al crecimiento (hemorragia, estenosis, intusucepción). No está indicada la linfadenectomía. El abordaje por laparoscopía permite una menor agresión parietal, menor dolor postoperatorio y un mejor resultado estético.

\section{CONCLUSIONES}

Si bien los schwannomas gástricos son extremadamente raros, el cirujano debe pensar en ellos cuando se enfrenta a un tumor submucoso gástrico. El estudio del mismo comparte el algoritmo de otros tumores submucosos gástricos, incluyendo la ecoendoscopía cuando esta está disponible. El tratamiento quirúrgico es la regla, idealmente por vía laparoscópica. El pronóstico a largo plazo es excelente cuando la resección es completa.

DECLARACIÓN DE CONFLICTOS DE INTERESES: Los autores no reportan ningún conflicto de interés. El estudio se realizó con recursos propios de los autores y/o la institución a la que representan.

\section{REFERENCIAS}

(1) Mazur MT, Clark HB. Gastric stromal tumors: reappraisal of histogenesis.

Am J Surg Pathol 1983; 7(6):507-19. doi: 10.1097/00000478-198309000-00001

(2) Daimaru Y, Kido H, Hashimoto H, Enjoji M. Benign schwannoma of the gastrointestinal tract: $A$ clinicopathologic and immunohistochemical study. Hum Pathol 1988; 19(3):257-64.

doi: 10.1016/s0046-8177(88)80518-5

(3) Voltaggio L, Murray R, Lasota J, Miettinen M. Gastric schwannoma: a clinicopathologic study of 51 cases and critical review of the literature. Hum Pathol 2012; 43(5):650-9.

doi: 10.1016/j.humpath.2011.07.006

(4) Couselo Villanueva JM, Arija Val F, Maseda Díaz O, Torres García MI, Guillán Millán R, Gegúndez Gómez C, et al. Schwannoma gástrico. Cir Esp 2000; 67(1):116-117.

(5) Yang JH, Zhang M, Zhao ZH, Shu Y, Hong J, Cao YJ. Gastroduodenal intussusception due to gastric schwannoma treated by billroth II distal gastrectomy: one case report.

World J Gastroenterol 2015; 21(7):2225-8.

doi: 10.3748/wjg.v21.i7.2225

(6) Lomdo M, Setti K, Oukabli M, Moujahid M, Bounaim A. Gastric schwannoma: a diagnosis that should be known in 2019.

J Surg Case Rep 2020; 2020(1):rjz382.

doi: $10.1093 /$ jscr/rjz382

(7) Ji JS, Lu CY, Mao WB, Wang ZF, Xu M.

Gastric schwannoma: CT findings and clinicopathologic correlation.

Abdom Imaging 2015; 40(5):1164-9. doi: $10.1007 / \mathrm{s} 00261-014-0260-4$

(8) Weisenberg E. Schwannoma. [Website]. [cited 14 sept 2019]. Available from: https://www.pathologyoutlines.com/topic/stomachschwannoma.html.

(9) Cabillón J, Estapé Viana G, Santiago P. Schwannoma Gástrico. Caso Clínico.

Cir Uruguay 2006; 76(2):157-164. 